\title{
Productivity losses impact cost effectiveness of COVID-19 vaccine
}

The cost effectiveness of COVID-19 vaccination is sensitive to vaccine price and productivity losses, according to findings of a Danish study published in Clinical Drug Investigation.

A dynamic transmission model developed by the Statens Serum Institut, under the Danish Ministry of Health, was used to evaluate the cost effectiveness of COVID-19 vaccination in older people ( $\geq 60$ years) and in younger people ( $<60$ years), from the perspective of the Denmark healthcare sector over a 6-month time horizon. Four scenarios were considered: vaccinating $25 \%$ of the total population but targeting people aged $\geq 60$ years; vaccinating $25 \%$ of the population but targeting people aged $<60$ years; vaccinating $40 \%$ of the population including $15 \%$ aged $<60$ years and $25 \%$ aged $\geq 60$ years; and vaccinating $40 \%$ of the population but targeting those aged $<60$ years. The assumed cost of vaccination ranged from DKK $300^{*}$ to DKK500 per fully vaccinated person.

At a $4 \%$ discount rate, the estimated incremental gain compared with no COVID-19 vaccination was 3480 QALYs with $25 \%$ vaccination of older people, 1270 with 25\% vaccination of younger people, 3670 QALYs with $15 \%$ and $25 \%$ vaccination of younger and older people, respectively, and 1640 QALYs with 40\% vaccination of younger people. Healthcare costs were lower when the younger population was targeted for vaccination than when the older population was targeted.

The estimated cost per life-year gained (LYG) was increased when people aged $\geq 60$ years only were targeted, or when both older and younger people were included. When productivity losses were not included, targeting people aged $\geq 60$ years dominated (more effective and less costly) targeting people aged $<60$ years only. However, if productivity losses were included and the vaccine price was DKK300, vaccinating $25 \%$ of people aged $<60$ years would become cost effective.

The estimated incremental cost-effectiveness ratio (ICER) was dependant on the price of the vaccine (including administration costs), and the discount rate used to estimate LYG or QALYs gained from vaccination.

"Without productivity losses, the elderly population should always be part of the target group for a COVID-19 vaccination programme ... Taking productivity losses into account, at least in case of low vaccine prices vaccinating the younger population first, can actually be cost-effective," concluded the authors.

* 2020 Danish kroner

Debrabant K, et al. The Cost-Effectiveness of a COVID-19 Vaccine in a Danish Context. Clinical Drug Investigation : 8 Oct 2021. Available from: URL: http:// doi.org/10.1007/s40261-021-01085-8 